\title{
Efficacité pollinisatrice de Apis mellifera (Hymenoptera : Apidae) sur Luffa cylindrica (L.) M. Roem (Cucurbitaceae) à Ngaoundéré (Cameroun)
}

\author{
Daniel FARDA* et Fernand-Nestor TCHUENGUEM FOHOUO
}

Laboratoire de Zoologie Appliquée, Faculté des Sciences, Université de Ngaoundéré, BP 454 Ngaoundéré, Cameroun.

*Auteur correspondant, E-mail: fardadaniel@gmail.com ; Tel : (+237) $675422271 / 690212123$.

\section{RESUME}

Afin d'évaluer l'impact de Apis mellifera sur les rendements fruitier et grainier de Luffa cylindrica, l'activité de butinage et de pollinisation des fleurs par cette abeille ont été étudiées à Dang. D'août à septembre 2013 et 2014, les expériences ont porté sur 440 fleurs femelles divisées en trois traitements : deux traitements différenciés selon la présence ou l'absence de protection des fleurs vis-à-vis des abeilles et d'autres insectes; le troisième traitement aux fleurs protégées puis ouverts au butinage par A. mellifera. Le comportement de butinage de cet insecte, son efficacité pollinisatrice, le taux de fructification et le pourcentage de graines normales ont été évalués. Les butineuses récoltaient intensément et régulièrement le nectar et le pollen. Le taux de fructification, le nombre de graines par fruit et le pourcentage de graines normales issues des traitements aux fleurs non protégées et visitées par les insectes sont de 94,58\%, 84,27 et 87,40\% respectivement. Pour les traitements aux fleurs protégées et visitées exclusivement par A. mellifera, les chiffres correspondants sont de $92,50 \%, 48,20$ et 79,96\%. L'installation des colonies de $A$. mellifera à proximité des populations de $L$. cylindrica est recommandée pour accroître les rendements en fruits et en graines et stabiliser les colonies d'abeilles en vue d'une bonne production du miel dans la région.

(C) 2018 International Formulae Group. All rights reserved.

Mots clés: Apis mellifera, Luffa cylindrica, fleurs, plante apicole, pollinisation, Dang.

\section{Pollination efficiency of Apis mellifera (Hymenoptera: Apidae) on Luffa cylindrica (L.) M. Roem (Cucurbitaceae) at Ngaoundere (Cameroon)}

\begin{abstract}
To evaluate the impact of Apis mellifera on fruit and seed yields of Luffa cylindrica, the foraging and pollinating activities of worker bees were studied at Dang. From August to September 2013 and 2014, the experiments were carried out mainly on 440 female flowers divided in three treatments: two treatments differentiated according to the presence or absence of protection regarding honeybee and other insect visits; the third protected and uncovered to allow honey bee visits. Bee's, foraging behavior on flowers, its pollination efficiency, the fruiting rate and percentage of normal seeds were evaluated. Workers bees intensely harvested nectar and pollen. The fruiting rate, the number of seeds per fruit and the percentage of normal seeds from treatment with unprotected flowers that were visited by insects were $94.58 \%, 84.27$ and $87.40 \%$ respectively. For the treatments with protected flowers that were visited by A. mellifera exclusively, the corresponding figures were $92.50 \%, 48.20$ and $79.96 \%$. The installation of A. mellifera colonies close to L. cylindrica
\end{abstract}


populations could be recommended to increase fruit and seed yields and to maintain honey bee colonies in view to improving honey production in the region.

(C) 2018 International Formulae Group. All rights reserved.

Keywords: Apis mellifera, Luffa cylindrica, flowers, bee plant, pollination, Dang.

\section{INTRODUCTION}

Les premières plantes à fleurs sont apparues au Bas Crétacé, il y a plus ou moins 130 millions d'années; depuis lors, elles se sont fortement diversifiées et constituent actuellement le groupe de plantes le plus répandu à la surface de la terre (Lejoly, 2005). Parmi ces plantes figure Luffa cylindrica, communément appelée éponge végétale, largement cultivée et commercialisée en Amérique central, Chine, Corée, Inde et au Japon (Bal et al., 2004). Les feuilles (Figure 1 A) de cette Cucurbitaceae sont utilisées pour combattre l'hémorroïde, l'hypertension artérielle et l'asthme; les jeunes fruits (Figure 1 D) sont comestibles (Sangh et al., 2012). L'éponge de L. cylindrica (Figure $1 \mathrm{E}$ ) entre également dans la confection de textiles " absorbants » tel que les serviettes éponges, les gants et les disquettes pour le nettoyage du visage (Oboh et Aluyor, 2009).

Selon Manjishtha et Susanta (2014), Luffa cylindrica est une plante herbacée annuelle monoïque se reproduisant à la suite d'une pollinisation croisée. Les feuilles vertes sont profondément découpées en lobes pointus plus ou moins dentelées persistant au bout d'un long pétiole pubescent (Oboh et Aluyor, 2009). La tige est dotée de longues vrilles qui s'enroulent à son extrémité et lui permettent de s'agripper sur les supports que constituent les branches d'arbres ou les tuteurs (Oboh et Aluyor, 2009). Les rameaux florifères portent des inflorescences mâles constituées de 5 à 7 fleurs chacune s'épanouissant en un seul jour (Figure $1 \mathrm{~B}$ ) et des fleurs femelles (Figure 1 C) solitaires (Oboh et Aluyor, 2009).

$\mathrm{Au}$ Cameroun, les productions fruitières et grainières de $L$. cylindrica sont faibles alors que leur demande par les utilisateurs est forte. Ce faible rendement peut être lié, d'une part, aux contraintes abiotiques de production (qualité du sol, faibles précipitations, températures élevées) et d'autre part à l'action de certains facteurs biotiques tels que les ravageurs, les agents pathogènes et l'absence ou l'insuffisance des pollinisateurs (Silué, 2009). Généralement, l'activité des pollinisateurs incluant de nombreux insectes est ignorée par la plupart des agriculteurs africains (Eardley et al., 2006). Pourtant, la productivité de diverses plantes cultivées dans le monde dépend en grande partie des insectes anthophiles qui en sont les principaux agents pollinisateurs (Stubbs et Drummond, 2001 ; Tchuenguem et al., 2002).

$\mathrm{Au}$ Cameroun, les relations entre quelques plantes et leurs insectes floricoles sont de plus en plus connues grâce aux recherches effectuées depuis plus de 28 ans dans les Régions de l'Ouest (Dongock et al., 2004), du Centre (Tchuenguem et al., 2002 ; Fomekong et al., 2008 ; Azo'o et al., 2010 ; Otiobo et al., 2015), de l'Adamaoua (Tchuenguem, 2005 ; 2010a ; Kingha et al., 2012; Mazi et al., 2014; Adamou et Tchuenguem, 2014 ; Népidé et Tchuenguem, 2016) et de l'Extrême-Nord (Azo'o et al., 2012a ; Douka et Tchuenguem, 2014 ; Dounia et Tchuenguem, 2014). Malgré ces importants travaux, il n'existe pas de données publiées sur l'interaction $L$. cylindrica et les insectes floricoles au Cameroun. Le présent travail est une contribution à la connaissance des relations entre L. cylindrica et A. mellifera, pour leur gestion optimale.

Les objectifs spécifiques inclus : a) l'étude de l'activité de A. mellifera sur les fleurs de L. cylindrica; b) l'estimation de la valeur apicole de cette Cucurbitaceae; c) l'évaluation de l'impact des insectes anthophiles sur les rendements de cette plante; d) la détermination de l'efficacité pollinisatrice de A. mellifera sur L. cylindrica. 


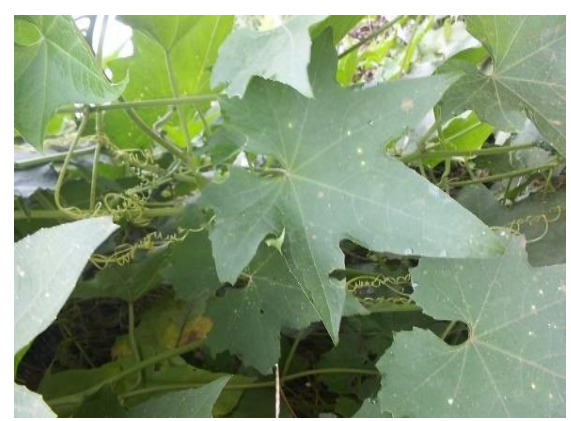

A

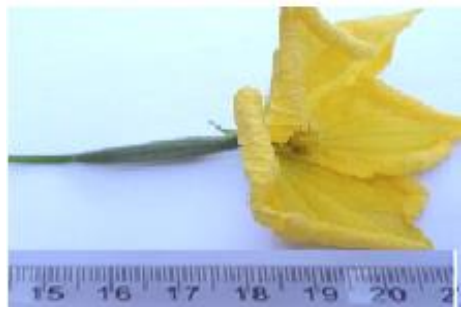

$\mathrm{C}$

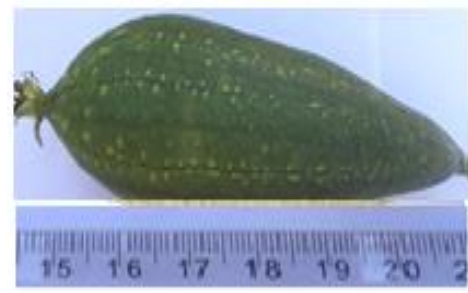

$\mathrm{D}$

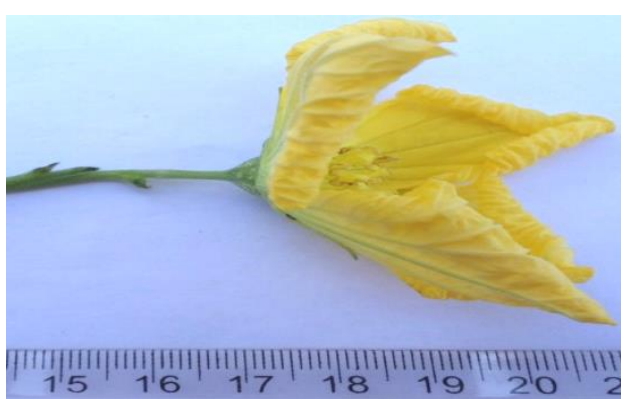

B

Figure 1: Pied (A), fleur mâle (B), fleur femelle (C), fruit (D) et éponge (E) de Luffa cylindrica.

\section{MATERIEL ET METHODES}

\section{Site et matériel biologique}

Les travaux ont été effectués du 16 mai 2013 au 09 novembre 2013 et du 08 mai 2014 au 04 novembre 2014 à Dang dans la région de l'Adamaoua-Cameroun, au sein du campus de l'Université de Ngaoundéré, sur une aire de $23 \mathrm{~m}$ de longueur sur $19 \mathrm{~m}$ de largeur centrée sur un point de latitude $7^{\circ} 25^{\prime} 26,42^{\prime} ' \mathrm{~N}$, de longitude $13^{\circ} 32^{\prime} 24,46^{\prime \prime} \mathrm{E}$ et d'altitude $1107,40 \mathrm{~m}$. Cette Région appartient à la zone écologique dite des hautes savanes guinéennes; le climat est de type soudanoguinéen, caractérisé par deux saisons: une saison des pluies (avril à octobre) et une saison sèche (novembre à mars). La pluviométrie annuelle est de $1500 \mathrm{~mm}$. La température moyenne annuelle est de $22^{\circ} \mathrm{C}$ et l'hygrométrie moyenne annuelle est de $70 \%$.

Pendant la période d'investigation, les fleurs de plusieurs autres espèces végétales du site d'étude étaient susceptibles d'attirer les butineuses de A. mellifera. Parmi ces plantes, les plus représentatives sont: a) trois Asteraceae: Cosmos sulphureus, Helianthus annuus et Tithonia diversifolia ; b) deux Fabaceae: Cajanus cajan et Phaseolus vulgaris; c) une Pedaliaceae: Sesamum indicum; d) deux Poaceae : Ctenium newtonii et Brachiara brizantha; e) une Malvaceae: Waltheria indica.

\section{Méthodes}

\section{Préparation de la parcelle expérimentale}

Du 15 avril au 05 mai 2013 et du 10 avril au 02 mai 2014, la parcelle expérimentale a été défrichée, labourée et huit sous - parcelles de $8 \mathrm{~m}$ de longueur, $4,5 \mathrm{~m}$ de largeur et $20 \mathrm{~cm}$ de hauteur chacune ont été formées. Les sous - parcelles étaient séparées les unes des autres par des allées de $1 \mathrm{~m}$.

\section{Semis et entretien de la culture}

Le semi a été fait le 09 mai 2013 et le 05 mai 2014 sur sous - parcelle, à raison de quatre lignes par sous - parcelle. Les graines ont été semées en poquet, à raison de cinq graines par poquet. L'écartement entre les poquets était de $150 \mathrm{~cm}$ sur les lignes et 200 $\mathrm{cm}$ entre les lignes. Deux semaines après la germination, le démariage a été effectué et deux plants les plus vigoureux ont été maintenus par poquet.

De la germination (survenue le 16 mai en 2013 et le 21 mai en 2014) à l'apparition de la première fleur ( 25 juillet en 2013 et 31 juillet en 2014), six opérations de sarclages ont été effectuées et les tuteurs de $65 \mathrm{~cm}$ de 
hauteur formés de deux fourches et d'une traverse ont été plantés sur chaque sous parcelle.

\section{Détermination du mode de reproduction de Luffa cylindrica}

Le 25 juillet 2013 et le 31 juillet 2014, 240 boutons floraux femelles ont été étiquetés sur les huit sous - parcelle (à raison de 55 boutons floraux par sous - parcelle) et deux traitements constitués :

- traitement 1: 120 boutons floraux libres ;

- traitement 2: 120 boutons floraux protégés des insectes à l'aide des sachets en toile gaze.

Dix jours après la fanaison de la dernière fleur étiquetée, le nombre de fruits formés dans chaque traitement a été compté. Pour chacun des traitements 1 et 2, l'indice de fructification (Ifr) a été calculé selon la formule ci - après (Tchuenguem et al., 2001) :

Ifr $=\left(F_{2} / F_{1}\right)$ où $F_{2}$ est le nombre de fruits formés et $F_{l}$ le nombre de fleurs viables initialement portées.

La différence entre les indices de fructification des deux traitements a permis d'apprécier les taux d'allogamie (TC) et d'autogamie (TA), selon les formules ci après (Demarly, 1977) :

$$
T C=\left[\left(I f r_{1}-I f r_{2}\right) / I f r_{1}\right] * 100 \text {, où } I f r_{1} \text { et }
$$

$I f r_{2}$ sont respectivement les indices de fructification dans le traitement aux fleurs laissées en libre pollinisation et dans le traitement aux fleurs protégées des insectes :

$$
T A=[100-T C] \text {. }
$$

\section{Etude de l'activité de Apis mellifera sur les fleurs de Luffa cylindrica}

Pour chaque année d'investigation, l'étude de la fréquence des visites a porté sur les fleurs du traitement 1. En ce qui concerne notamment l'étude de la durée des visites par fleurs, de la vitesse de butinage et de l'abondance par 1000 fleurs, plusieurs fleurs mâles ont en outre été considérées.

Les observations ont été effectuées tous les jours, du 26 août 2013 au 7 septembre 2013, puis du $1^{\text {er }}$ au 14 septembre 2014, pendant cinq tranches horaires journalières : 6
$-7 \mathrm{~h}, 8-9 \mathrm{~h}, 10-11 \mathrm{~h}, 12-13 \mathrm{~h}$ et $14-15$ h. Il était question de passer une fois sur chacune des fleurs du traitement 1 et pour chacune de ces tranches horaires. A chaque passage, les différents insectes rencontrés sur les fleurs épanouies étaient comptés. Les insectes n'ayant pas été marqués, les résultats cumulés ont été exprimés par le nombre de visites (Tchuenguem, 2005) Les données obtenues ont permis de déterminer la fréquence des visites de $A$. mellifera sur les fleurs femelles de L. cylindrica. La fréquence des visites $\left(F_{i}\right)$ de l'insecte ${ }_{i}$ sur les fleurs de L. cylindrica est calculée à l'aide de la formule suivante :

$$
F_{i}=\left[V_{i} / V_{I} * 100\right] \text {, avec } V_{i}=\text { nombre de }
$$

visites de l'insecte $i$ sur les fleurs du traitement aux fleurs laissées en libre pollinisation et $V_{I}$ le nombre de visites de tous les insectes sur ces mêmes fleurs (Tchuenguem et al., 2004). Les données sur la fréquence relative des visites des différents insectes anthophiles recensés ont permis de déterminer la fréquence relative de $A$. mellifera dans l'entomofaune floricole de $L$. cylindrica (Tchuenguem et al., 2004).

Les produits floraux prélevés étaient systématiquement notés lors de l'enregistrement de la durée des visites par fleur. Il s'agissait de noter si sur une fleur, l'abeille récolte le pollen, le nectar ou ces deux aliments. Une butineuse qui plonge sa trompe dans une fleur est une récolteuse de nectar; si à l'aide des mandibules et des pattes elle gratte les anthères, il s'agit d'une récolteuse de pollen (Jean-Prost, 1987).

La durée d'une visite est le temps que met une butineuse de A. mellifera pour prélever un produit floral (nectar et / ou pollen) sur une fleur (Kearns et Inouye, 1993). Les durées des visites de récolte du pollen et celles de prélèvement du nectar ont été enregistrées séparément. Le chronomètre (marque PC 1000; sensibilité 1/1000 de seconde) ramené à zéro est mis en marche dès qu'une butineuse se pose sur une fleur et arrêté dès le départ de celle - ci. La durée de cette visite correspond à la valeur lue sur le chronomètre (Tchuenguem et al., 2004). Ce paramètre a été enregistré pendant les mêmes 
dates pour la fréquence des visites, selon cinq tranches horaires journalières $(7-8 \mathrm{~h}, 9-10$ $\mathrm{h}, 11-12 \mathrm{~h}, 13-14 \mathrm{~h}$ et $15-16 \mathrm{~h}$ ), à raison d'au moins cinq valeurs par produit florale et par tranche horaire, lorsque l'activité des butineuses le permettait.

Les abondances par fleur ont été enregistrées à la suite des comptages directs. Pour l'abondance par 1000 fleurs $\left(A_{1000}\right)$, les individus de chaque espèce d'insecte ont été comptés sur un nombre connu de fleurs épanouies. L'abondance par 1000 fleurs $\left(A_{1000}\right)$ a ensuite été calculée à l'aide de la formule suivante : $A_{1000}=\left[\left(A_{x} / F_{x}\right) * 1000\right]$, où $F_{x}$ et $A_{x}$ sont respectivement le nombre de fleurs épanouies et le nombre d'insectes butineurs effectivement comptés sur les fleurs laissées en libre pollinisation à l'instant $x$ (Tchuenguem et al., 2004). Les données ont été enregistrées aux mêmes dates et tranches horaires que pour la durée des visites par fleur, à raison d'au moins cinq valeurs par tranche horaire, lorsque l'activité de cette abeille le permettait.

La vitesse de butinage (nombre des fleurs visitées par minute: Jacob-Remacle, 1989) a été enregistrée. Le chronomètre mis à zéro était déclenché dès qu'une butineuse se posait sur une fleur. Le nombre de fleurs visitées par celle-ci était alors compté, au fur et à mesure qu'elle passait de fleur en fleur. Le chronomètre était stoppé dès que la butineuse était perdue de vue. La vitesse de butinage $\left(V_{b}\right)$ est : $V_{b}=(F i / d i) * 60$ où $d i$ la durée donnée par le chronomètre (en seconde) et $F i$ est le nombre de fleur correspondant à $d i$ (Tchuenguem et al., 2004).

Le nombre de visites interrompues par des compétiteurs ou prédateurs lors de l'activité de butinage de A. mellifera et l'attractivité des fleurs des plantes avoisinantes ont été systématiquement notée, lors du chronométrage de la durée des visites par fleur.

Tout au long de la période d'observation, la température et l'hygrométrie de la station d'étude ont été enregistrées de 6 $\mathrm{h}$ à $18 \mathrm{~h}$, toutes les 30 minutes, par un thermo - hygromètre portable instablé à l'ombre (Tchuenguem et al., 2004).
Estimation de la valeur apicole de Luffa cylindrica

Comme dans d'autres travaux (Villières, 1987 ; Karanja et al., 2010 ; Mensah et Kudom, 2011), la valeur apicole de L. cylindrica a été évaluée à l'aide principalement des données sur son intensité de floraison et l'attractivité des ouvrières de A. mellifera vis - à - vis du nectar et du pollen.

\section{Etude des rendements de Luffa cylindrica}

$\mathrm{Au}$ terme de la floraison, les fruits ont été comptés dans chaque traitement et les taux de fructification calculés. Pour un traitement ${ }_{X}$, le taux de fructification $\left(F_{x}\right)$ est :

$F_{x}=\left[\left(F r_{f} / F l_{p}\right) * 100\right]$ où $F r_{f}$ est le nombre de fruits formés et $F l_{p}$ le nombre de fleurs initialement portées

A la maturité des fruits, la récolte a été faite, puis le nombre total de graines par fruit et le nombre de graines normales (c'est-à-dire bien développées) par fruit ont été comptés. Les données obtenues ont permis de calculer, le nombre moyen de graines par fruit et le pourcentage de graines normales pour chacun des traitements où cela était possible.

\section{Evaluation de l'impact des insectes anthophiles dont Apis mellifera sur les rendements de Luffa cylindrica \\ Comme dans d'autres travaux} (Tchuenguem et al., 2009a; 2009b; Azo'o et al., 2010 ; Kudom et Kwapong, 2010), elle est basée sur: l'impact des insectes anthophiles sur la pollinisation; l'impact de la pollinisation sur la fructification; la comparaison des rendements fruitiers (taux de fructification) du traitement aux fleurs laissées en libre pollinisation à ceux du traitement aux fleurs protégées des insectes (Tchuenguem et al., 2001).

Pour chaque année d'étude, le taux de fructification $\left(P_{i}\right)$ dû à l'influence des insectes floricoles a été calculé à l'aide de la formule suivante (Tchuenguem et al., 2004) :.

$$
P_{i}=\left[\left(F_{1}-F_{2}\right) / F_{1}\right] * 100 \text { où } F_{1} \text { et } F_{2}
$$
sont les taux de fructification dans les traitements 1 (fleurs laissées en libre pollinisation) et 2 (fleurs protégées) respectivement. 


\section{Estimation de l'efficacité pollinisatrice de Apis mellifera sur Luffa cylindrica}

Parallèlement à la mise en place des traitements 1 et 2 , un troisième traitement a été mis en place. Il était formé de 200 boutons floraux femelles étiquetés et protégés comme ceux du traitement 2 et dont les fleurs épanouies étaient destinées au butinage par $A$. mellifera exclusivement. Dès l'épanouissement de chaque fleur, la toile gaze était délicatement enlevée pendant les périodes d'activités optimales des abeilles et les fleurs laissées en libre pollinisation observée pendant une à 10 minutes, pour noter sa visite éventuelle par A. mellifera. Après cette manipulation, la fleur était de nouveau protégée (Fameni et al., 2012).

L'estimation de l'efficacité pollinisatrice de A. mellifera sur L. cylindrica est basée sur la comparaison des rendements fruitiers (taux de fructification) des traitements 2 et 3 .

Le taux de fructification dû à l'efficacité pollinisatrice spécifique de $A$. mellifera $\left(P_{a}\right)$ a été calculé à l'aide de la formule ci-après :

$$
P_{a}=\left[\left(F_{2}-F_{3}\right) / F_{2}\right] * 100 \text {, où } F_{2} \text { et } F_{3}
$$

sont les taux de fructification dans les traitements 2 et 3 respectivement (Tchuenguem et al., 2004).

\section{Traitement des données}

Le logiciel Excel 2007 a été utilisé pour trois tests : Test - $t$ de Student au seuil de $5 \%$ pour la comparaison des moyennes de deux échantillons; Chi - carré $\left(\chi^{2}\right)$ pour la comparaison des pourcentages ; Coefficient de corrélation de Pearson $(r)$ pour l'étude des relations linéaires entre deux variables.

\section{RESULTATS}

\section{Mode de reproduction de Luffa cylindrica}

En 2013, l'indice de fructification moyen a été de $0,94(n=120 ; s=0,24)$ dans le traitement 1 et $0(n=120 ; s=0)$ dans le traitement 2. En 2014 cet indice a été de 0,95 $(\mathrm{n}=120 ; \mathrm{s}=0,21)$ dans le traitement 1 et 0 ( $\mathrm{n}$ $=120 ; \mathrm{s}=0$ ) dans le traitement 2 . Ainsi, en 2013 comme en $2014, T C=100 \%$ et $T A=$ $0 \%$. En conséquence, la variété de $L$. cylindrica étudiée a un mode de reproduction exclusivement allogame.

Activité de Apis mellifera sur les fleurs de Luffa cylindrica

Fréquence des visites

En 2013 et 2014 respectivement, 286 et 418 visites de six et huit espèces d'insectes ont été dénombrées sur 120 fleurs femelles de L. cylindrica. Le Tableau 2 présente la liste de ces insectes avec leurs fréquences de visites. Il ressort de ce Tableau 2 que parmi les insectes floricoles de L. cylindrica, A. mellifera a été le plus fréquemment observé, avec $61,19 \%$ et $42,34 \%$ de visites en 2013 et 2014 respectivement. La différence entre ces deux pourcentages est très hautement significative $\left(\chi^{2}=24,12 ; d d l=1 ; P<0,001\right)$.

\section{Produits floraux prélevés}

Au niveau des fleurs femelles de $L$. cylindrica, les ouvrières de A. mellifera ont récolté intensément du nectar (Figure 3), seul produit florale disponible. Dans les fleurs mâles, ces abeilles récoltaient fortement le nectar et le pollen (Figure 4). En 2013, sur 1814 visites chronométrées sur les fleurs staminées, 957 (soit 52,75\%) étaient consacrées à la récolte du nectar et 857 (soit $47,24 \%$ ) pour le prélèvement du pollen. Pour l'année 2014, sur 1170 visites, les valeurs correspondantes étaient de 626 (soit 53,50\%) pour le nectar et 544 (soit $46,49 \%$ ) pour le pollen.

\section{Rythme des visites selon les étapes de la floraison}

Dans l'ensemble, les visites d'abeilles étaient plus nombreuses dans le champ de $L$. cylindrica quand le nombre de fleurs épanouies était plus élevé (Figure 5). La corrélation entre le nombre de fleurs épanouies et le nombre de visites de $A$. mellifera s'est révélée positive et très hautement significative, aussi bien en 2013 ( $r$ $=0,98 ; d d l=11 ; P<0,001)$ qu'en $2014(r=$ $0,99 ; d d l=12 ; P<0,001)$.

\section{Abondance des butineuses}

En 2013, le nombre moyen d'ouvrières de A. mellifera observées en activité au même moment a été de 1,38 par fleur de $L$. cylindrica $(n=188 ; s=0,63)$; l'abondance 
moyenne était de 111 butineuses par 1000 fleurs $(n=188 ; s=32,85)$. En 2014, les chiffres correspondants étaient de 1,36 par fleur $(n=145 ; s=0,65)$ et 137 par 1000 fleurs $(n=145 ; s=33,48)$. La différence entre les abondances moyennes par 1000 fleurs n'est pas significative $(t=2,55 ; d d l=$ $331 ; P>0,01)$.

\section{Durée des visites par fleur}

En 2013, la durée moyenne d'une visite de $A$. mellifera sur une fleur femelle de L. cylindrica était de 13,91 $\mathrm{sec}(n=1116 ; s=$ 16,72) avec un temps maximal de $158 \mathrm{sec}$; Sur les fleurs mâles, cette durée moyenne était de $8,84 \sec (n=957 ; s=8,14)$ avec un temps maximal de $65 \mathrm{sec}$ pour la récolte du nectar et de $9,11 \mathrm{sec}(n=857 ; s=11,58)$ avec un temps maximal de $93 \mathrm{sec}$ pour la récolte du pollen. La différence entre la durée moyenne d'une visite de récolte du nectar dans une fleur femelle et une fleur mâle est très hautement significative $(t=194,01 ; d d l=$ $2071 ; P<0,001)$; cette différence est très hautement significative entre cette même durée moyenne d'une visite de récolte du nectar dans une fleur femelle et une fleur mâle $(t=158 ; d d l=1971 ; P<0,001)$.

Pour l'année 2014, sur une fleur femelle, la durée moyenne d'une visite de récolte du nectar de cette abeille était de 13,37 sec $(n=1002 ; s=17,05)$ avec un temps maximal de $188 \mathrm{sec}$ et de $8,23 \mathrm{sec}(n=543 ; s$ $=10,04)$ avec un temps maximal de $71 \mathrm{sec}$ dans une fleur mâle; pour le prélèvement du pollen, cette durée était de 10,36 $\mathrm{sec}$ ( $n=$ $625 ; s=12,23$ ) avec un temps maximal de $158 \mathrm{sec}$. La différence entre la durée moyenne d'une visite de récolte du nectar dans une fleur femelle et une fleur mâle est très hautement significative $(t=121,07 ; d d l=$ 1542 ; $P<0,001)$; cette différence est très hautement significative entre cette même durée moyenne d'une visite de récolte du nectar dans une fleur femelle et une fleur mâle ( $t=74,91 ; d d l=1624 ; P<0,001)$.

\section{Vitesse de butinage}

Sur la parcelle de L. cylindrica, en 2013, une butineuse de A. mellifera visitait en moyenne 8,87 fleurs/min pour la récolte de nectar $(n=389 ; s=6,10)$ et 12,05 fleurs/min pour la récolte de pollen ( $n=248 ; s=12,24)$. En 2014, les chiffres correspondant étaient 8,12 fleurs/min pour la récolte du nectar $(n=$ $238 ; s=4,65)$ et 11,07 fleurs/min pour la récolte de pollen $(n=176 ; s=10,78)$. La différence entre la vitesse moyenne de butinage du nectar et celle de la récolte du pollen est très hautement significative aussi bien en 2013 ( $t=-53,41 ; d d l=635 ; P<$ $0,001)$ qu'en $2014(t=-37,86 ; d d l=412 ; P<$ 0,001).

\section{Influence de la faune}

Les visites de $A$. mellifera étaient perturbées par d'autres insectes qui étaient des concurrents pour la recherche du nectar et/ou de pollen ou des prédateurs. Les interruptions de visites avaient lieu à la suite des collisions entre les visiteurs, des tentatives de capture ou de l'approche d'une fleur déjà occupée par un autre visiteur. Ainsi, sur 1116 visites chronométrées en 2013 au niveau des fleurs femelles, 127 (soit 11,37\%), 59 (soit 5,28\%), et 24 (soit 2,15\%) étaient interrompues respectivement par Myrmicaria opaciventrice, Xylocopa olivacea et A. mellifera et; en 2014, sur 1002 visites des fleurs femelles, 56 (soit $5,58 \%$ ), 6 (soit 0,59\%), et 4 (soit 0,59\%) étaient interrompues respectivement par Myrmicaria opaciventrice, Xylocopa olivacea et A. mellifera.

Au niveau des fleurs mâles, sur 1814 visites enregistrées en 2013, 87 (soit 4,79\%), 53 (soit 2,92\%), et 47(soit 2,59\%), étaient interrompues respectivement par Myrmicaria opaciventrice, Xylocopa olivacea et $A$. mellifera; en 2014, sur 1170 visites chronométrées, 53 (soit 4,52\%), 27 (soit $2,30 \%$ ), et 10 (soit $0,85 \%$ ), étaient interrompues respectivement par Myrmicaria opaciventrice, Xylocopa olivacea, et A. mellifera. Pour obtenir leur charge maximale de nectar et/ou de pollen, les butineuses qui subissent de telles perturbations sont obligées de visiter un plus grand nombre de fleurs et/ou de plantes lors du voyage de butinage correspondant.

\section{Influence de la flore avoisinante}

Au cours de la période de floraison de L. cylindrica, plusieurs autres espèces végétales situées à proximité du champ 
expérimental étaient aussi visitées par $A$. mellifera, pour leur pollen (po) et/ou leur nectar (ne). Parmi ces plantes, il y a Cosmos sulphureus (ne et po), Helianthus annuus (ne et po), Tithonia diversifolia (ne et po), Cajanus cajan (ne et po), Phaseolus vulgaris (ne et po), Sesamum indicum (ne et po), Ctenium newtonii (po), Brachiara brizantha (po) et Waltheria indica (ne et po).

Lors des voyages de butinage, nous n'avons noté aucun passage de A. mellifera des fleurs de L. cylindrica aux fleurs d'autres espèces végétales et vice versa. Les butineuses de A. mellifera étaient ainsi fidèles aux fleurs de L. cylindrica lors des voyages de butinage.

\section{Influence de quelques facteurs climatiques}

Pour les 13 journées d'observation de la Cucurbitaceae en 2013, huit étaient fortement ensolleillées, cinq brumeuses et pluvieuses. Pour les 14 jours d'observation de 2014, dix étaient ensolleillées, quatre brumeuses et pluvieuses. Pendant les journées ensoleillées, l'abondance des butineuses sur les fleurs de la plante était élevée. Lors des journées brumeuses et pluvieuses, l'activité de ces abeilles mellifères était faible ou nulle.

La Figure 6 montre les variations de la température et de l'hygrométrie de la station d'étude puis du nombre de visites de $A$. mellifera selon les tranches horaires journalières d'observation en 2013 et en 2014. Les analyses statistiques montrent qu'il n'y a pas de corrélation entre la température et le nombre de visites de A. mellifera en 2013 ( $r=$ $0,23 ; d d l=4 ; P>0,05)$ comme en 2014 ( $r=$ - 0,34; ddl = 4;P>0,05). De même, la corrélation entre l'hygrométrie et le même nombre de visites de A. mellifera n'est pas significative en $2013(r=-0,01 d d l=4 ; P>$ $0,05)$ comme en $2014(r=0,10 ; d d l=4 ; P>$ $0,05)$.

\section{Valeur apicole de Luffa cylindrica}

Pendant les périodes de floraison de $L$. cylindrica en 2013 et 2014, l'activité remarquable des ouvrières de $A$. mellifera aux niveaux des fleurs était noté. En particulier, il y avait une grande fréquence des visites, une bonne récolte de nectar dans les fleurs mâles comme dans celles femelles, un fort prélèvement de pollen au niveau des fleurs mâles et une fidélité des butineuses aux fleurs lors des voyages de butinage. Par ailleurs chaque pied de L. cylindrica peut produire 200 à plus de 500 fleurs femelles et 300 à plus de 600 fleurs mâles, s'épanouissant au tour de $6 \mathrm{~h}$ et tombent progressivement à partir de $12 \mathrm{~h}$ jusqu'à $16 \mathrm{~h}$. Ces observations mettent en évidence la forte attractivité du nectar et du pollen de L. cylindrica vis - à - vis de $A$. mellifera. Elles permettent de classer $L$. cylindrica parmi les plantes apicoles fortement nectarifères et pollinifères.

\section{Impact de Apis mellifera sur la pollinisation de Luffa cylindrica}

Au moment de la récolte du pollen et / ou du nectar, les ouvrières de A. mellifera se trouvaient fréquemment en contact avec le stigmate (fleurs femelles) et avec les anthères (fleur mâles), comme l'indique le Tableau 2. Il ressort de ce tableau que les fréquences des contacts entre A. mellifera et le stigmate lors de la récolte du nectar dans les fleurs femelles étaient de 97,57\% et 95,57\%, en 2013 et 2014 respectivement; pour le prélèvement du nectar dans les fleurs mâles, les fréquences des contacts avec les anthères étaient de $100 \%$ en 2013 et $100 \%$ en 2014 . Les butineuses de A. mellifera transportaient le pollen de $L$. cylindrica (à l'aide notamment des pièces buccales, des pattes et de la fourrure) de fleur en fleur sur une ou sur plusieurs plantes. Ces abeilles pouvaient, en conséquence, intervenir dans la pollinisation croisée, en mettant le pollen d'une fleur sur le stigmate d'une autre fleur de la même plante ou en mettant le pollen d'une fleur sur le stigmate d'une fleur d'une autre plante.

\section{Rendements de Luffa cylindrica}

Le Tableau 3 présente le taux de fructification, le nombre moyen de graines par fruit et le pourcentage de graines normales (Figure 7) dans les différents traitements de $L$. cylindrica. Il ressort de l'exploitation des données du Tableau 3 que pour les deux années d'investigation cumulées :

- les fleurs femelles protégées des insectes n'ont produit aucun fruit ;

- le taux de fructification, le nombre de graine par gousse et le pourcentage de graines normales issus des traitements aux fleurs 
femelles non protégées et visitées par les insectes dont A. mellifera sont de 94,58\%, 84,27 et $87,40 \%$ respectivement ;

- le taux de fructification, le nombre de graine par gousse et le pourcentage de graines normales issus des traitements aux fleurs femelles protégées et visitées exclusivement par A. mellifera sont de $92,50 \%, 48,20$ et $79,96 \%$.

La comparaison deux à deux des chiffres du Tableau 3 montre que la différence :

a) n'est pas significative entre les taux de fructification des traitements 1 et $3\left(\chi^{2}=\right.$ $0,77 ; d d l=1 ; P>0,05) ; b)$ est très hautement significative entre les nombres moyens de graines par fruit des traitements 1 et $3(t=5372,17 ; d d l=20458 ; P<0,001)$; c) n'est pas significative entre les pourcentages de graines normales des traitements 1 et $3\left(\chi^{2}=0,79 ; d d l=1 ; P>\right.$ $0,05)$. Ainsi, entre les traitements 4 et 6 , la différence : a) n'est pas significative entre les taux de fructification $\left(\chi^{2}=0,30 ; d d l=1 ; P>\right.$ $0,05) ; b$ ) est très hautement significative entre les nombres moyens de graines par fruit $(t=$ $4022,31 ; d d l=17488 ; P<0,001)$; c) est très hautement significative entre les pourcentages de graines normales $\left(\chi^{2}=523,77 ; d d l=1 ; P\right.$ $<0,001)$.

Impact des insectes floricoles dont Apis mellifera sur les rendements de Luffa cylindrica

Le taux de fructification, dû à l'influence des insectes anthophiles a été de 100\% en 2013 comme en 2014.

Efficacité pollinisatrice de Apis mellifera sur Luffa cylindrica

Le taux de fructification attribuable à l'activité de A. mellifera a été de $100 \%$ en 2013 comme en 2014.

Tableau 1: Insectes recensés sur les fleurs femelles de Luffa cylindrica à Dang en 2013 et 2014, nombre et pourcentage de visites des différents insectes.

\begin{tabular}{|c|c|c|c|c|c|c|c|c|}
\hline \multicolumn{3}{|r|}{ Insectes } & \multicolumn{2}{|c|}{2013} & \multicolumn{2}{|c|}{2014} & \multicolumn{2}{|c|}{ Total $_{2013 / 2014}$} \\
\hline Ordre & Famille & Genre, Espèce & $\mathrm{n}_{1}$ & $\mathrm{P}_{1}(\%)$ & $\mathrm{n}_{2}$ & $\mathrm{P}_{2}(\%)$ & $\mathrm{n}_{\mathrm{T}}$ & $\mathrm{P}_{\mathrm{T}}(\%)$ \\
\hline \multirow{8}{*}{$\begin{array}{l}\text { Hymenopte } \\
\text { ra }\end{array}$} & & & & & 17 & & & \\
\hline & Apidae & Apis mellifera $(\mathrm{Ne})$ & 175 & 61,19 & 7 & 42,34 & 352 & 49,37 \\
\hline & & Xylocopa olivacea $(\mathrm{Ne})$ & 45 & 15,73 & 40 & 9,57 & 95 & 13,32 \\
\hline & & Xylocopa inconstans $(\mathrm{Ne})$ & 6 & 2,10 & 27 & 6,46 & 33 & 4,63 \\
\hline & & Dactylurina staudingerii $(\mathrm{Ne})$ & - & - & 53 & 12,68 & 53 & 7,43 \\
\hline & & Meliponula ferruginea $(\mathrm{Ne})$ & - & - & 43 & 10,29 & 43 & 6,03 \\
\hline & Formicida & & & & & & & \\
\hline & $\mathrm{e}$ & Myrmicaria opaciventrice $(\mathrm{Ne})$ & 56 & 19,58 & 71 & 16,98 & 127 & 17,81 \\
\hline Coleoptera & Meloidae & Coryna sp. (mange les fleurs) & 1 & 0,35 & - & - & 1 & 0,14 \\
\hline \multirow[t]{3}{*}{ Lepidoptera } & Pieridae & Eurema eximia $(\mathrm{Ne})$ & 3 & 1,06 & 4 & 0,96 & 7 & 0,98 \\
\hline & & Eurema sp. $(\mathrm{Ne})$ & - & - & 3 & 0,72 & 3 & 0,42 \\
\hline & & & & & 41 & & & \\
\hline Total & & & 286 & 100 & 8 & 100 & 713 & 100 \\
\hline Espèces & & & & 6 & & 8 & & 9 \\
\hline
\end{tabular}

sp.: espèce indéterminée ; $\mathrm{n}_{1}:$ nombre de visites sur 120 fleurs en 13 jours, $\mathrm{n}_{2}:$ nombre de visites sur 120 fleurs en 14 jours ; $\mathrm{P}_{1}$ et $\mathrm{P}_{2}$ : pourcentages de visites, $\mathrm{P}_{1}=\left(\mathrm{n}_{1} / 286\right) * 100,\left(\mathrm{P}_{2}=\left(\mathrm{n}_{2} / 418\right) * 100 ; \mathrm{n}_{\mathrm{T}}\right.$ : nombre total de visites; $\mathrm{P}_{\mathrm{T}}$ : pourcentages total de visites; $\mathrm{Ne}$ : récolte du nectar.

Comparaison des pourcentages de visites de Apis mellifera (2013/2014): $\chi^{2}=24,12, \mathrm{ddl}=1, \mathrm{P}<0,001$. 
Tableau 2: Nombre et la fréquence des contacts entre Apis mellifera et le stigmate (fleurs femelles) ou les anthères (fleurs mâles) lors de la récolte du nectar chez L. cylindrica en 2013 et 2014 à Dang.

\begin{tabular}{ccccccc}
\hline $\begin{array}{c}\text { Types } \\
\text { de fleur }\end{array}$ & Années & $\begin{array}{c}\text { Nombre de } \\
\text { visites } \\
\text { étudiées }\end{array}$ & \multicolumn{2}{c}{$\begin{array}{c}\text { Visites avec contact abeille- } \\
\text { stigmate }\end{array}$} & \multicolumn{2}{c}{$\begin{array}{c}\text { Visites avec contact abeille- } \\
\text { anthères }\end{array}$} \\
\cline { 3 - 7 } & & Nombre & Pourcentage (\%) & Nombre & Pourcentage (\%) \\
\hline Femelle & 2013 & 1116 & 1085 & $97,57 \%$ & - & - \\
& 2014 & 1002 & 947 & $95,57 \%$ & - & - \\
\multirow{2}{*}{ Mâle } & 2013 & 957 & - & - & 141 & $100 \%$ \\
& 2014 & 544 & - & - & 131 & $100 \%$ \\
\hline
\end{tabular}
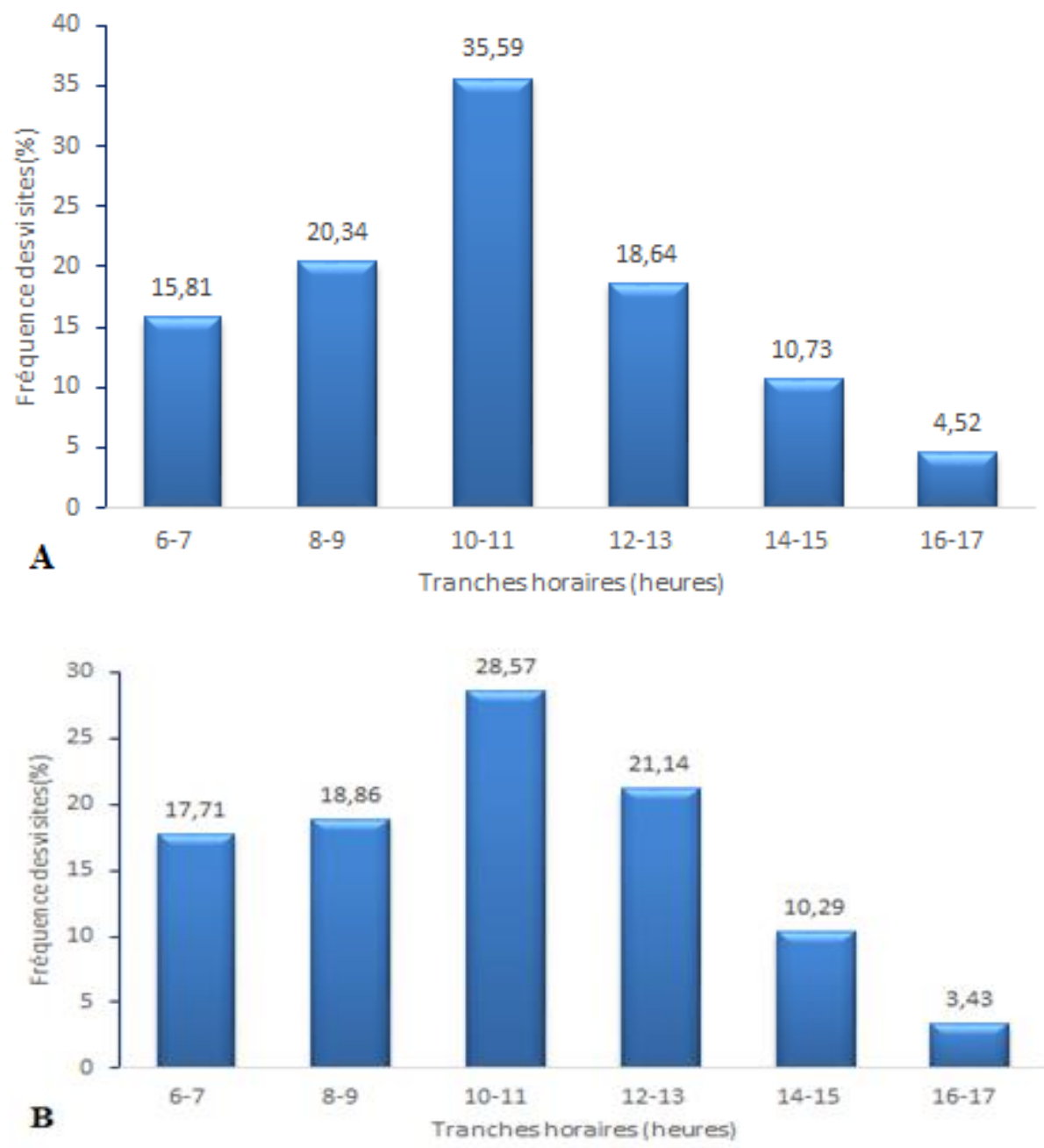

Figure 2: Fréquence de visites d'Apis mellifera sur 120 fleurs femelles de Luffa cylindrica selon les tranches horaires journalières d'observation en 2013 (A) et 2014 (B) à Dang. 


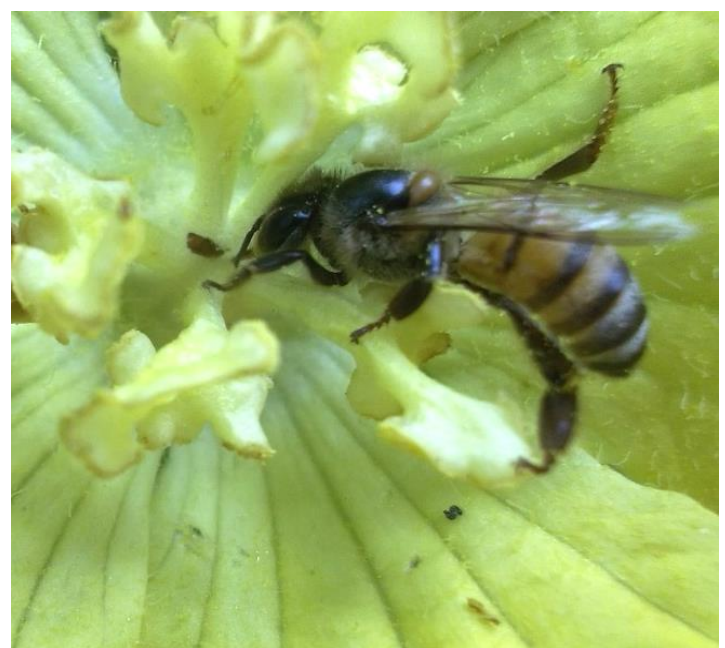

Figure 3: Ouvrière de Apis mellifera récoltant le nectar dans une fleur femelle de Luffa cylindrica

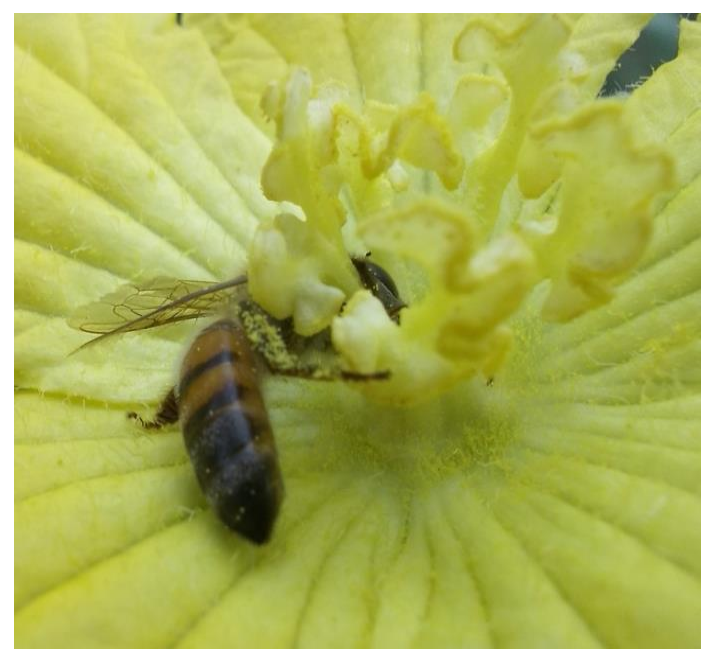

Figure 4 : Ouvrière de Apis mellifera récoltant le pollen dans une fleur mâle de Luffa cylindrica

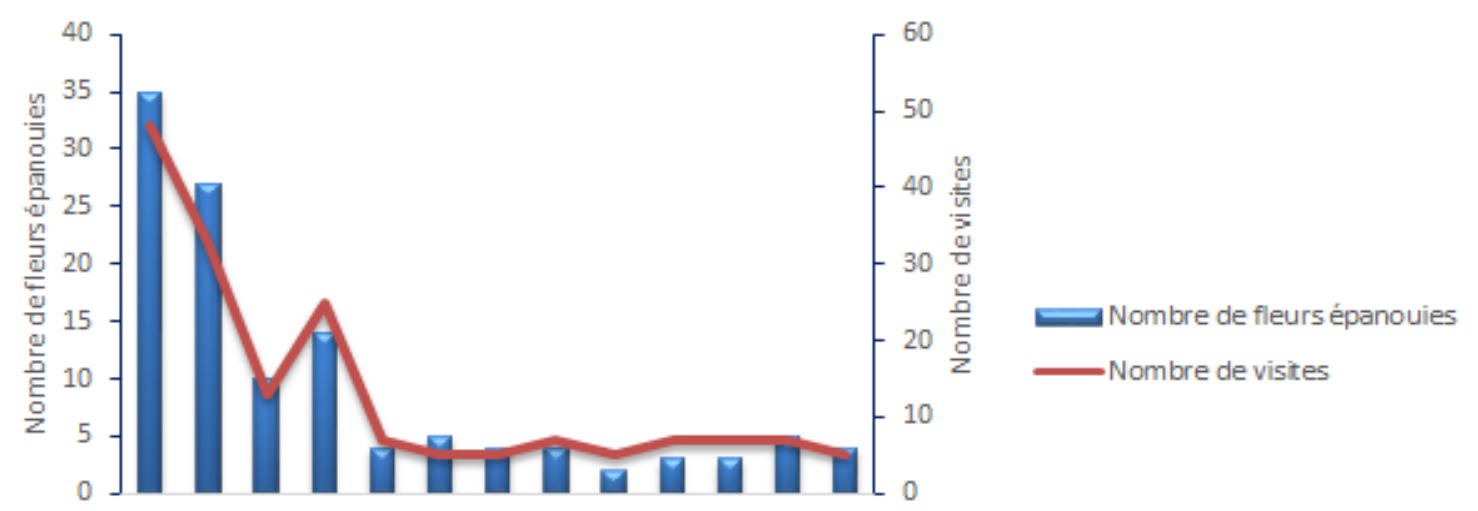

A

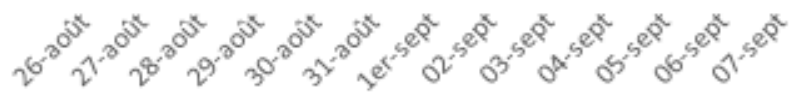

Dates (2013)

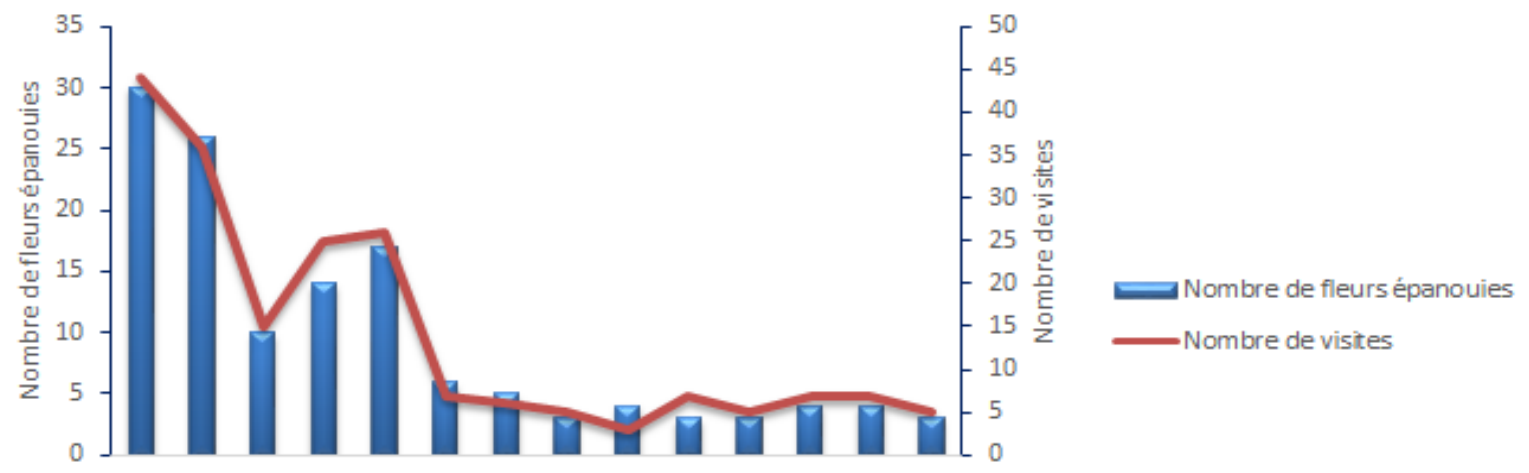

B

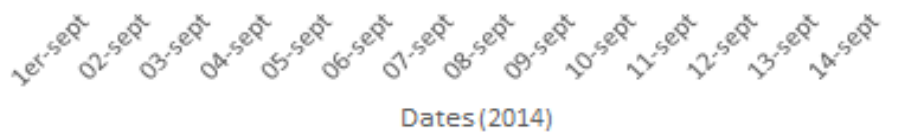

Figure 5: Variation du nombre de fleurs épanouies et du nombre de visites de Apis mellifera sur les fleurs de Luffa cylindrica selon les dates d'observation en 2013 (A) et en 2014 (B) à Dang. 


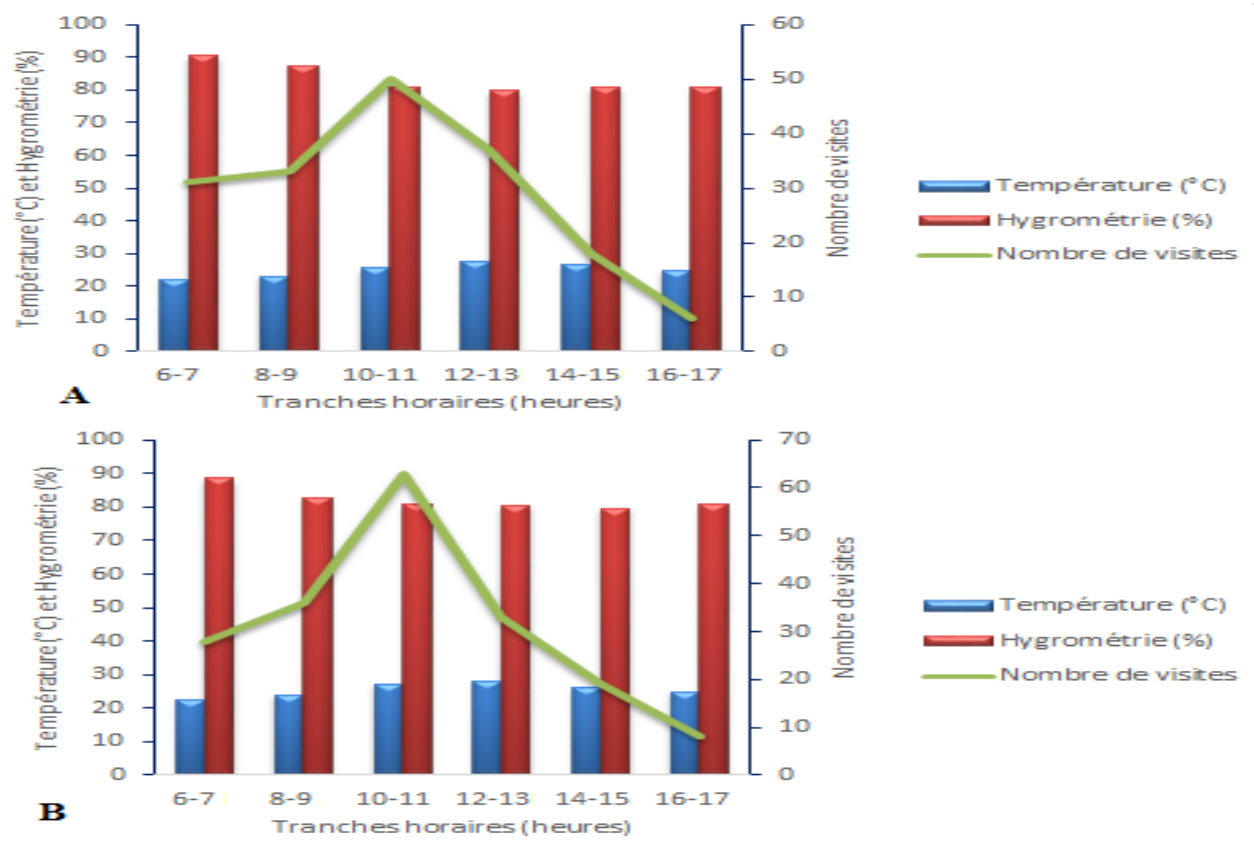

Figure 6: Variation journalière de la température, de l'humidité relative de l'air et du nombre de visites de Apis mellifera sur les fleurs de Luffa cylindrica en 2013 (A) et en 2014 (B) à Dang.

Tableau 3: Taux de fructification, nombre moyen de graines par fruit et pourcentage de graines normales selon les traitements de Luffa cylindrica en 2013 et 2014 à Dang.

\begin{tabular}{llllllllll}
\hline Années & Traitements & NFE & NFF & \multirow{2}{*}{ TF(\%) } & \multicolumn{2}{c}{ Graines/fruit } & \multirow{2}{*}{ NTG } & NGN & PGN \\
\cline { 6 - 7 } & & & & & $\boldsymbol{m}$ & $\boldsymbol{s}$ & & & \\
\hline 2013 & $\mathbf{1}($ Flp) & 120 & 113 & 94,17 & 95,43 & 43,35 & 11651 & 9767 & 85,29 \\
& 2 (Fp) & 120 & 0 & 0 & - & - & - & - & - \\
& 3 (FpA) & 200 & 183 & 91,5 & 52,82 & 34,51 & 10564 & 8809 & 83,38 \\
\hline 2014 & $\mathbf{4}$ (Flp) & 120 & 114 & 95 & 73,11 & 39,53 & 8773 & 7854 & 89,52 \\
& $\mathbf{5}$ (Fp) & 120 & 0 & 0 & - & - & - & - & - \\
& 6 (FpA) & 200 & 187 & 93,5 & 43,58 & 22,24 & 8717 & 6672 & 76,54 \\
\hline
\end{tabular}

Flp: fleurs femelles laissées en libre pollinisation ; Fp : fleurs femelles protégées ; FpA : fleurs femelles pollinisées par $A$. mellifera, NFE : nombre de fleurs $£$ étudiées, NFF : nombre de fruits formés, TF : taux de fructification, $m:$ moyenne; $s$ : écart-type, NTG : nombre total de graines, NGN : nombre de graines normales, PGN : pourcentage de graines normales.

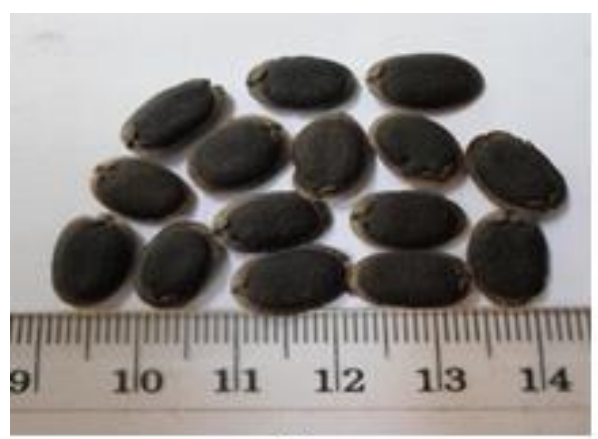

A

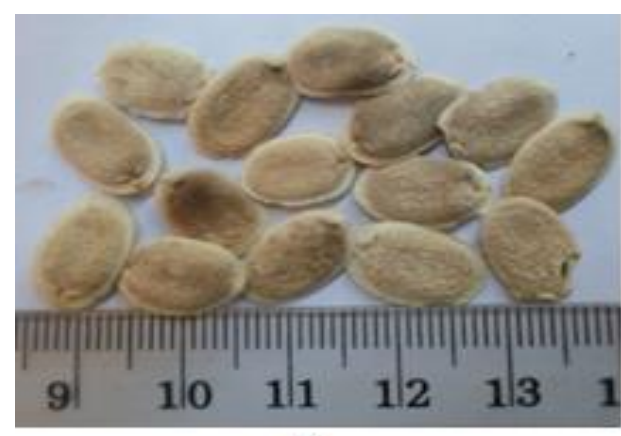

B

Figure 7: Graines normales (A) et graines anormales (B) de Luffa cylindrica. 


\section{DISCUSSION}

\section{Activité de Apis mellifera sur les fleurs de Luffa cylindrica}

A Dang, Apis mellifera est le principal insecte floricole de L. cylindrica par contre, au Ghana, Mensah et Kudom (2011) signalent que Xylocopa olivacea est l'insecte le plus fréquent sur les fleurs d'une autre espèce de Luffa (L. aegyptiaca). Ces différences pourraient être dues à la faible présence des populations de A. mellifera au niveau des stations d'investigation du Ghana. Les abeilles Apis mellifera sont connues comme faisant partie des insectes floricoles les plus fréquents sur autres Cucurbitaceae dont Cucumeropsis mannii (Fomekong et al., 2008), Citrullus lanatus (Azo'o et Messi, 2012b).

Le nombre très élevé des visites de $A$. mellifera par rapport à celui des autres insectes floricoles s'expliquerait par la stratégie de butinage optimale qui caractérise cette abeille. Comme l'ont signalé Fomekong et al. (2008) sur Cucumeropsis mannii, ce recrutement aurait certainement pour conséquence la limitation du nombre de visites des autres espèces d'insectes due à l'occupation de la majorité des fleurs épanouies par A. mellifera.

Le pic d'activité de A. mellifera sur les fleurs de $L$. cylindrica a été mis en évidence dans la matinée, entre $10 \mathrm{~h}$ et $11 \mathrm{~h}$. Cette période de la journée correspondrait au moment de plus grande disponibilité du nectar et/ou du pollen au niveau des fleurs de la Cucurbitacée. Il est, en effet, connu que l'activité quotidienne de butinage des abeilles domestiques sur les fleurs d'une plante dépend de sa production de pollen et/ou de nectar (Suzo et al., 2001 ; Pouvreau, 2004).

La forte abondance des butineuses de A. mellifera par 1000 fleurs et la corrélation positive et significative entre le nombre de visites de cette abeille et le nombre de fleurs épanouies de $L$. cylindrica mettent en évidence l'attractivité élevée du nectar et/ou du pollen de cette Cucurbitaceae vis-à-vis de l'abeille mellifère.

Chez L. cylindrica, le pollen est produit par les anthères qui sont situées au- dessus des étamines, et sont, de ce fait, facilement accessibles à l'abeille, alors que le nectar se trouve dans le tube corollaire, entre la base du style et des étamines et est difficilement accessible.

Les interruptions de visites avaient pour conséquence la réduction de la durée de certaines visites. Ceci obligeait certaines ouvrières à visiter un plus grand nombre de fleurs lors d'un voyage de butinage, afin d'obtenir leur charge maximale de nectar et/ou de pollen. (Tchuenguem et al., 2007).

L'absence de passages de A. mellifera de L. cylindrica à plusieurs autres espèces végétales prouve que cette abeille est fidèle aux fleurs de la Cucurbitacée lors des voyages de butinage. Ce phénomène est dénommé «constance florale» (Basualdo et al., 2000; Montgomery, 2009). Ladite constance florale s'explique par le fait que chez Apis mellifera, la butineuse est en général capable de mémoriser et de reconnaître la forme, la couleur et l'odeur des fleurs visitées lors des voyages de butinages antérieurs (Wright et al., 2002 ;). La fidélité de cette abeille a été démontrée sur les fleurs de plusieurs autres plantes dont Helianthus annus (Basualdo et al., 2000, Tchuenguem et al., 2009), Lycopersicum esculentum (Sabara et Winston, 2003), Vitellaria paradoxa (Djonwanwé et al., 2011), Oxalis barrelieri (Otiobo et al., 2015), Croton macrostachyus (Népidé et Tchuenguem, 2016)

Luffa cylindrica peut être cultivé et protégé pour accroître la production du miel et du pollen comme produit de la ruche, puisque son nectar et son pollen attirent fortement les butineuse de A. mellifera, comme l'a également noté Usman (2013) au Nigéria. Cette plante a été également répertoriée comme plante mellifère au Benin (Akoegnignou et al., 2010). En tant que plante apicole très fortement nectarifère et pollinifère, la Cucurbitaceae L. cylindrica peut également contribuer efficacement à l'alimentation et donc au maintien des colonies d'abeille pendant la saison des pluies. 
Impact de l'activité de $\boldsymbol{A}$. mellifera sur la pollinisation et les rendements de Luffa cylindrica.

Du fait que l'abeille A. mellifera est régulièrement en contact avec le stigmate lors de la récolte du nectar sur les fleurs femelle et avec les anthères lors de la récolte du pollen sur les fleurs mâles, elle augmente fortement les possibilités de pollinisation de $L$. cylindrica. Apis mellifera transporte le pollen de fleur en fleur sur L. cylindrica. Elles peuvent, en conséquence, transporter le pollen d'une fleur d'un pied de la plante donnée sur le stigmate d'une autre fleur de la même plante (geitonogamie) ou mettre ce pollen sur le stigmate d'une fleur produit par un autre individu (xénogamie) (Djonwanwé et al., 2011).

L'intervention de A. mellifera dans la pollinisation de L. cylindrica est d'autant plus probable que sa densité par 1000 fleurs et sa vitesse de butinage sont particulièrement élevées et que la pollinisation entomophile croisée est obligatoire chez cette plante (Manjishtha et Susanta, 2014). Ce dernier argument explique l'absence de fruits dans le traitement aux fleurs protégées des insectes pendant toute la durée de leur épanouissement, aussi bien en 2013 qu'en 2014. La contribution positive et significative de A. mellifera dans la fructification de $L$. cylindrica se justifie ainsi par l'action des butineuses sur l'autopollinisation et la pollinisation croisée. Plusieurs fleurs visitées par A. mellifera n'ont pas données de fruits. Ce résultat démontre que nombreuses visites d'insectes sur les fleurs de L. cylindrica sont bénéfiques a ces arthropodes mais n'ont aucune influence sur la pollinisation et les rendements de la Cucurbitacée.

\section{Conclusion}

A Dang, la pollinisation de $L$. cylindrica est strictement entomogame. Parmi les insectes qui visitent les fleurs de cette plante, A. mellifera est de loin le plus fréquent et récolte fortement le nectar et le pollen. La comparaison des rendements des fleurs laissées en libre pollinisation à ceux des fleurs protégées des insectes met évidence une production de fruits due exclusivement aux insectes pollinisateurs dont $A$. mellifera. Par le biais de son efficacité pollinisatrice, $A$. mellifera a provoqué une fructification très élevée des fleurs visitées. L'installation et/ou le maintien des colonies de A. mellifera à proximité des champs de L. cylindrica est recommandée dans l'Adamaoua pour accroître la production des fruits et des graines et maintenir les populations de A. mellifera en vue d'une bonne production ultérieure de miel.

\section{CONFLIT D'INTERETS}

Aucun conflit d'intérêt à notre connaissance n'a été signalé entre les auteurs.

\section{CONTRIBUTION DES AUTEURS}

\section{REFERENCES}

Adamou M, Tchuenguem FFN. 2014. Foraging and pollination behavior of Apis mellifera adansonii Latreille (Hymenoptera, Apidae) on Brachiaria brizantha (Hochst. Ex A. Rich.) Stapf. 1919 flowers at Dang (NgaoundereCameroon). Int. J. Agron. Agric. Res., 4(6): $\quad$ 62-74. DOI : http://www.innspub.net

Akoegninou A, Tossou MG, Yedomonhan H, Fohounfo TH, Traore D. 2010. Etude des plantes mellifères de la petite saison des pluies et de quelques aspects du comportement des abeilles au Benin. Ann. Bot. Afr. Ouest, 6: 1-13. DOI : http://www.scirp.org/(S(Iz5mqp453edsn p55rrgjct55))/reference/

Azo'o EM, Messi J. 2012a. Yield responses of Cucumeropsis mannii (Cucurbitaceae) to the presence or the absence of the insect foraging activity at Nkolbisson in Cameroon. J. Anim. Plant Sci., 13(3): 1791-1799. DOI: http://www.m. elewa.org/JAPS

Azo'o EM, Madi A, Tchuenguem FFN, Messi J. 2012b. The importance of a single floral visit of Eucara macrognatha and Tetralonia fraterna (Hymenoptera: Apidae) in the pollination and the yields of Abelmoschus esculentus in Maroua, 
Cameroon. Afr. J. of Agrc. Res., 7(18): 2853-2857. DOI: http://dx.doi.org/ 10.5897/AJAR12.359

Azo'o EM, Messi J, Tchuenguem FFN, Tamesse LJ, Kekenou S, Pando JB. 2010. Foraging behavior of Apis mellifera adansvonii and its impact on pollination, fruit and seeds yields of Citrullus lanatus at Nkolbisson (Yaoundé, Cameroon). Cam. J. Exp. Biol., 6(1): 41-48. DOI: http://dx.doi.org/ 10.4314/cajeb. 656879

Bal KJ, Hari BKC, Radha KT, Madhusudan G, Bhuwon RS, Madhusudan PU. 2004. Descriptors for sponge gourd [Luffa cylindrica (L.) Roem.J. Nepal Agricultural Research Council (NARC), Kathmandu Nepal, Local Initiatives for Biodiversity, Research and Development (LIBIRD). Pokhara Nepal and International Plant Genetic Resources Institute (IPGRI): Rome; 43.

Basualdo M, Bedascarrabure E, De jong D. 2000. Africanized honey bees (Hymenoptera: Apidae) have a greater fidelity to sunflowers than European bees. J. Econ. Entomol., 93: 304-307. DOI :

http//bibliotecavirtual.clacso.org.ar/ar/lib ros/grupos/basua/C04/Basualdo.pdf

Demarly 1977. Génétique et Amélioration des Plantes. Masson : Paris ; 577 p.

Djonwangwé D, Tchuenguem FFN, Messi J, Brückner D. 2011. Impact de l'activité de butinage de Apis mellifera adansonii Latreille (Hymenoptera : Apidae) sur la pollinisation et la chute des jeunes fruits du karité Vitellaria paradoxa (Sapotaceae) à Ngaoundéré (Cameroun). Int. J. Biol. Chem. Sci., 5(4): 1538-1551. DOI: http://ajol.info/index.php/ijbcs

Dongock ND, Foko J, Pinta JY, Ngouo LV, Tchoumboue J, Zango p. 2004. Inventaire des plantes mellifères de la zone soudano-guinéenne d'altitude de l'Ouest Cameroun. Tropicultura, 22(3): 139-145. DOI : http//www. researchgate. net/publication/45266396

Douka C, Tchuenguem FFN. 2014. Foraging and pollination activity of Musca domestica L. (Diptera : Muscidae) on flowers of Ricinus communis L. (Euphorbiaceae) at Maroua, Cameroon. J. Biod. Environ. Sci., 4(3): 63-76. DOI: http://www.

10.1.1.654.1888\&rep=rep1\&type=pdf

Dounia, Tchuenguem FFN. 2014. Foraging and pollination activity of Macronomia vulpina (Gerstaecker, 1857) (Hymenoptera: Halictidae) on Gossypium hirsutum L. (Malvaceae) flowers at Maroua, Cameroon. Int. J. Agron. Agric. Res., 4(3): 1-10. DOI: http://www.innspub.net

Eardley C, Roth D, Clarke J, Buchmann S, Gemmill B. 2006. Pollinators and pollination: A Resource Book for Policy and Practice. African Pollinator Initiative; 77.

Fameni TS, Tchuenguem FFN, Brückner D. 2012. Pollination efficiency of Apis mellifera adansonii (Hymenoptera: Apidae) on Callistemon rigidus (Myrtaceae) flowers at Dang (Ngaoundere, Cameroon). Int. J. Trop. Insect Sci., 32(1): 2-11. DOI: http://www.10.1017/S174275841200003 3

Fomekong A, Messi J, Kekeunou S, Tchuenguem FFN, Tamesse JL. 2008. Entomofauna of Cucumeropsis mannii Naudin, its impact on plant yield and some aspects of the biology of Dacus bivitattus (Diptera : Tephritidae). Afr. J. Agric. Res., 3(5): 363-370. DOI : http://www.academicjournals.org/AJAR

Jacob-Remacle A. 1989. Comportement de butinage de l'abeille domestique et des abeilles sauvages dans des vergers de pommiers en Belgique. Apidologie, 20 : 271-285. DOI : http://www. 10.1051/apido:19890401

Jean-Prost P. 1987. Apiculture : Connaître l'abeille - Conduire le Rucher $\left(6^{\text {̀̀me }}\right.$ édn). Lavoisier : Paris ; 579.

Karanja RHN, Njoroge GN, Gikungu MW, Newton JKLE. 2010. Bee interactions with wild flora around organic and conventional coffee farms in Kiambu district. Cent. Agric. Technol., 4(5): 34- 
38. $\quad$ DOI : http://www. pollinationecology.org/

Kearns CA, Inouye DW. 1993. Techniques for pollination biologists. University Press of Colorado, Niwot, p. 583.

Kingha TBM, Tchuenguem FFN, Ngakou A, Brückner D. 2012. Foraging and pollination activities of Xylocopa olivacea (Hymenoptera, Apidae) on Phaseolus vulgaris (Fabaceae) flowers at Dang (Ngaoundere - Cameroon). $J$. Agric. Ext. Rural Dev., 4(6): 330-339. DOI: http://www. 10.5897/JAERD11.151

Kudom AA, Kwapong PK. 2010. Floral visitors of Ananas comosus in Ghana: A preliminary assessment. J. Pollinat. Ecol., 2(5): 27-32. http://www.beekeeping.or.jp/wordpress/ wp-content/uploads/2013/10/H25pollinator-report.pdf

Lejoly 2005. Systématique des plantes à fleurs en relation avec les principales plantes médicinales. Institut de Pharmacie, Université Libre de Bruxelles: Bruxelles ; 295.

Manjishtha B, Susanta KC. 2014. Luffa cylindrica as a host plant for pollinator bees - a study based in West Midnapore, West Bengal. J. Entomol. Zool. Stud., 2(3): 21-26. DOI: http://www.entomoljournal.com

Mazi S, Tchuenguem FFN, Brückner D. 2014. Foraging and pollination behaviour of Chalicodoma rufipes L. (Hymenoptera: Megachilidae) on Cajanus cajan L. Mill sp. (Fabaceae) flowers at Dang (Ngaoundéré, Cameroon). Int. J. Agron. Agric. Res., 4(4): 77-88. DOI: http://www.innspub.net

Mensah BA, Kudom AA. 2011. Foraging dynamics and pollination efficiency of Apis mellifera and Xylocopa olivacea on Luffa aegyptiaca Mill (Cucurbitaceae) in Southern Ghana. J. Pollinat. Ecol., 4(5): 34-38. DOI: http://scholar.google.com/ citations

Montgomery BR. 2009. Do pollen carryover and pollinator constancy mitigate effects of ompetition for pollination? Oikos,
118: $\quad 1084-1092 . \quad$ DOI

https ://doi.org/10.1111/j.1600-

0706.2009.17064.x

Népidé NC, Tchuenguem FFN. 2016. Pollination efficiency of Apis mellifera adansonii Latreille (Hymenoptera : Apidae) on Croton macrostachyus (Euphorbiaceae) flowers at Dang, Ngaoundere, Cameroon. Int. J. Biosci., 9(3): $\quad 75-88 . \quad$ DOI: http://dx.doi.org/10.12692/ijb/9.3.75-88

Oboh IO, Aluyor EO. 2009. Luffa cylindrica an emerging cash crop. Afr. J. Agric. Res., 4(8): 684-688. DOI : http://www.academicjournals.org/AJAR

Otiobo AEN, Tchuenguem FFN, DjiétoLordon C. 2015. Activité de butinage et de pollinisation de Apis mellifera adansonii Latreille (Hymenoptera : Apidae) sur les fleurs d'Oxalis barrelieri (Oxalidaceae) à Yaoundé (Cameroun). Entomol. Faun., 68: 101 - 108. DOI: www.entomoljournal.com

Pouvreau A. 2004. Les Insectes Pollinisateurs. Delachaux and niestlé : Paris ; $157 \mathrm{p}$.

Sangh P, Saurabh K, Amit K, Sharma NK, Jha KK. 2012. In-Vitro Anthelmintic Activity of Luffa cylindrica Leaves in Indian Adult Earthworm. $J$. Pharmacogn. Phytochem., 1(2): 27-30. DOI: www.phitojournal.com

Saraba HA, Winston ML. 2003. Managing honey bees (Hymenoptera: Apidae) for greenhouse tomato pollination. J. Econ. Entomol., 98: 367-372. DOI : http://www.ncbi.nlm.nih.gov/m/pubmed/ $12852587 /$

Silué S. 2009. Mécanismes génétiques de l'embryogenèse chez Phaseolus et application en hybridation interspécifique. Thèse de Doctorat. Faculté Universitaire des Sciences Agronomiques de Gembloux, p. 140.

Stubbs CA, Drummond FA. 2001. Bombus impatiens (Hymenoptera: Apidae): an alternative to Apis mellifera (Hymenoptera: Apidae) for lowbush blueberry pollination. J. Econ. Entomol., 94(3): 609-616. DOI : 
https://ncbi.nlm.gov/m/pubmed/1142501 3/

Suzo MJ, Pierre J, Moreno MT, Esnault R, Le Guen J. 2001. Variation in outcrossing levels in faba bean cultivars: role of ecologycal factors. J. Agric. Sci., 136: 399-405. DOI : https://doi.org/10.1017/ s0021859601008851

Tchuenguem FFN, Messi J, Pauly A. 2001. Activité de Meliponula erythra sur les fleurs de Dacryodes edulis et son impact sur la fructification. Fruits, 56: 179-188. DOI : www.interesjournals.org

Tchuenguem FFN. 2005. Activité de butinage et de pollinisation de Apis mellifera adansonii Latreille (Hymenoptera : Apidae, Apinae) sur les fleurs de trois plantes à Ngaoundéré (Cameroun) : Callistemon rigidus (Myrtaceae), Syzygium guineense var. macrocarpum (Myrtaceae) et Voacanga africana (Apocynaceae). Thèse de Doctorat d'Etat, Université de Yaoundé I, p. 103.

Tchuenguem FFN, Djonwangwé D, Messi J, Brückner D. 2009a. Activité de butinage et de pollinisation de Apis mellifera adansonii Latreille (Hymenoptera : Apidae, Apinae) sur les fleurs de Helianthus annuus (Asteraceae) à Ngaoundéré (Cameroun). Cam. J. Exp. Biol., 5(1): 1-9.

Tchuenguem FFN, Fameni TS, Mbianda PA, Messi J, Brückner D. 2010a. Foraging behaviour of Apis mellifera adansonii (Hymenoptera : Apidae) on Combretum nigricans, Erythrina sigmoidea, Lannea kerstingii and Vernonia amygdalina flowers at Dang (Ngaoundéré, Cameroon). Int. J. Trop. Insect Sci., 30: 40-47. DOI : http://www.10.1017/S174275841000001 9

Tchuenguem FFN, Messi J, Pauly A. 2002. L'activité de butinage des Apoïdes sauvages (Hymenoptera: Apoidea) sur les fleurs de maîs à Yaoundé (Cameroun) et réflexions sur la pollinisation des graminées tropicales. Biotechnol., Agron., Soc. Environ., 6(2): 87-98. DOI : http://www. ingentaconnect.com

Tchuenguem FFN, Messi J, Brückner D, Bouba B, Mbofung G, Hentchoya HJ. 2004. Foraging and pollination beaviour of the African honey bee (Apis mellifera adansonii) on Callistemon rigidus flowers at Ngaoundéré (Cameroon). J. Cam. Acad Sci., 4: 133 - 140. DOI: http://scholar.googleusercontent.com

Tchuenguem FFN, Ngakou A, Kengni BS. 2009b. Pollination and yield responses of cowpea (Vigna unguiculata L. Walp.) to the foraging activity of Apis mellifera adansonii (Hymenoptera: Apidae) at Ngaoundéré (Cameroon). Afr. J. Biotechnol., 8(9): 1988-1996. DOI: http://www.academicjournals.org/AJB and http://cajeb.ifrance.com

Tchuenguem FFN, Djonwangwe D, Messi J, Brückner D. 2007. Expoitation des fleurs de Entada africana, Eucalyptus camaldulensis, Psidium guajava et Trichillia emetica par Apis mellifera adansonii à Dang (Ngaoundéré, Cameroun). Cam. J. Exp. Biol., 3(2): 5060. DOI: http://cajeb.ifrance.com

Usman HD. 2013. Identification of plants visited by the honeybee, Apis mellifera $L$. in the Sudan Savanna zone of northeastern Nigeria. Afr. J. Plant Sci., 7(7): 273-284. DOI: http://www.academicjournals.org/AJPS

Villières B. 1987. L'apiculture en Afrique Tropicale. Dossier «Le Point Sur $n^{\circ}$ 11. GRET, Paris ; 220.

Wright GA, Skinner BD, Smith BH. 2002. Ability of honeybee, Apis mellifera, to detect and discriminate odors of varieties of canola (Brassica rapa and Brassica napus) and snopgragon flowers (Antirrhinum majus). J. Chem. Ecol., 28(4): $\quad 721-740 . \quad$ DOI : https//books.google.cm 